\title{
In the non-cirrhotic stage of nonalcoholic steatohepatitis, angioarchitecture of portal veins and lobular architecture are maintained
}

\author{
Hiroshi Hano • Satoshi Takasaki • Hirohiko Kobayashi • \\ Tomoki Koyama • Tomoe Lu • Keisuke Nagatsuma \\ Received: 5 December 2012 /Revised: 7 March 2013 / Accepted: 15 March 2013 / Published online: 28 March 2013 \\ (C) The Author(s) 2013. This article is published with open access at Springerlink.com
}

\begin{abstract}
The morphogenesis of lobular restructuring to liver cirrhosis in nonalcoholic steatohepatitis (NASH) is yet to be clearly understood. Therefore, we observed tissue samples from three biopsies and one autopsy with NASH in the noncirrhotic stage three-dimensionally to elucidate the evolution of fibrosis and the changes of angioarchitecture. Histologic reconstructions revealed that pericellular fibrosis developed around the central vein in the early stage and gradually progressed to arch-shaped band-like fibrosis connecting the central veins in the neighboring lobules. In contrast, the basic angioarchitecture of the portal vein in the portal tracts tended to be preserved in the non-cirrhotic stage, although the portal vein architecture was slightly altered as the portal tract underwent gradual fibrous expansion. In addition, a striking development of arteries originating from the portal tract was found in the fibrotic area around the central and sublobular veins. In summary, while central-central bridging fibrosis and ectopic arterial development were conspicuous, the lobular architecture was maintained relatively well in the noncirrhotic stage of NASH because of only mildly damaged angioarchitecture of the portal veins. The process of lobular restructuring in NASH is considered to be different from that in chronic viral hepatitis in the non-cirrhotic stage.
\end{abstract}

H. Hano $(\bowtie) \cdot$ S. Takasaki $\cdot$ T. Lu

Department of Pathology, Jikei University School of Medicine,

3-25-8 Nishi-Shinbashi,

Minato-ku, Tokyo 105-8461, Japan

e-mail: hhano@jikei.ac.jp

H. Kobayashi $\cdot$ K. Nagatsuma

Internal Medicine Division of Gastroenterology and Hepatology,

Jikei University School of Medicine, 3-25-8 Nishi-Shinbashi,

Minato-ku, Tokyo 105-8461, Japan

T. Koyama

Department of Surgery, Jikei University School of Medicine,

3-25-8 Nishi-Shinbashi,

Minato-ku, Tokyo 105-8461, Japan
Keywords Nonalcoholic steatohepatitis · Histologic reconstruction - Three-dimensional observation .

Angioarchitecture $\cdot$ Lobular restructuring

\section{Introduction}

Nonalcoholic steatohepatitis (NASH) is characterized by histological features strikingly similar to those in alcoholic hepatitis [1-7]. It has recently been shown that patients with NASH have insulin resistance in the peripheral tissue in common $[8$, 9]. NASH seems to be increasing and has become an increasingly important liver disease worldwide. For a diagnosis of NASH, histological examination of liver biopsy is essential because NASH diagnosis is primarily histological [10-12]. To date, there have been many reports on the pathological findings which indicate that NASH leads to liver cirrhosis and occasionally to liver cell carcinoma [13-16]. A grading and staging system for necroinflammatory activity (grade) and fibrosis (stage) for NASH was proposed to assess the histologic features $[17,18]$. This system is generally accepted as being reproducible. However, it has not been fully understood how fibrosis evolves in NASH and results in the development of cirrhosis, in which also restructuring of liver lobules is involved. We therefore observed liver tissues with NASH in the non-cirrhotic stage three-dimensionally, focusing on the evolution of fibrosis and the change of angioarchitecture in connection with lobular restructuring, to answer the question whether these aspects are different in the non-cirrhotic stage of NASH and chronic viral hepatitis.

\section{Materials and methods}

Of fifty-six liver samples diagnosed as NASH collected in our department, three biopsies and one autopsy tissue 
sample were selected because they were suitable for the aim of this study and the preparation of serial sections. The tissue blocks were fixed in $10 \%$ formalin and embedded in paraffin. Sections were stained with HE, Masson's trichrome, PAS, and silver impregnation for histopathological evaluation. In addition, serial sections were cut at 3 . $5 \mu \mathrm{m}$ and stained with Masson's trichrome for threedimensional observation by a tissue reconstruction method. For tissue reconstruction, microscopical images captured with the aid of a computer were printed, and the outlines of essential architectural elements were drawn serially in freehand on sheets of tracing paper. The reconstruction was completed by step-by-step overlapping of the outlines.

Henceforth, we use the terminology and concepts defined by Matsumoto et al. with reference to the angioarchitecture of the normal liver $[19,20]$. The outline of this concept is as follows. The portal system is divided into the conducting and parenchymal portions. The main function of the former is the transportation of blood, while the latter provides the angioarchitectural framework of the liver lobule in addition to blood transportation. The portal veins of the parenchymal portion comprise the first, second, and third step branches. The first step branches arise directly from the terminal conduction portion. The second step branches originate approximately at a right angle from the first step branches and correspond to the portal veins in the portal tracts located in the periphery of the classical hexagonal lobule. The third step branches originate from the second step branches and run with a limited connective tissue sheath along the boundary zone of adjacent lobules.

This study was approved by the Ethics Committee of the Jikei University School of Medicine.

\section{Results}

Clinical data and histologic staging following the Brunt system of the four cases are shown in Table 1. None of the patients had a history of alcohol abuse and serological findings indicating hepatitis $\mathrm{B}$ or $\mathrm{C}$.
The first case was a 36-year-old obese Japanese man. He had been followed up because of fatty liver and mild hyperlipidemia for 13 years. He underwent liver biopsy upon suspicion of NASH because the serum transaminase level fluctuated above $100 \mathrm{IU} / 1$.

Histological features were relatively uniform throughout the specimen (Fig. 1a, b). The basic lobular structure was fairly well maintained. Moderate macrovesicular steatosis and mild delicate pericellular fibrosis had developed around central veins. Bridging fibrosis was absent. Foci of necroinflammation with lymphocytic and/or neutrophilic infiltration and lipogranuloma were sparsely found in the lobules. Ballooning cells appeared in groups. Mallory-Denk bodies were not observed. The portal tracts were fairly well preserved.

Histological reconstruction of the two regions was carried out using a total of 100 sections (together about $350 \mu \mathrm{m}$ thickness). The light microscopic features corresponding to the first reconstructed region are shown in Fig. 1a. The reconstruction contained central and sublobular veins and fairly well-preserved portal tract with mild fibrosis and mild lymphocytic infiltration (Fig. 1c). A remarkable change was pericellular fibrosis spreading out irregularly, mainly along the central and terminal portions. A few fine terminal portions were buried in the loose network of pericellular fibrosis (arrows in Fig. 1c). The branching pattern of the portal vein remained almost normal in the portal tract, which almost undamaged. The third step branches ran into the sinusoid to perfuse the parenchyma. No bridging fibrosis was seen. The basic structure of the liver lobules was fairly well preserved. The histology of the second reconstructed area was basically similar to that of the first one (Fig. 1b). The histologic reconstruction is shown in Fig. 1d. Irregular pericellular fibrosis developed around the central vein and terminal portions and buried them partially (arrows in Fig. 1d). The branching pattern of the portal vein showed close resemblance to that in the normal portal tract, indicating that at this relatively early stage, the portal areas were hardly damaged.

The second case was a 37-year-old Japanese woman with obesity and hyperlipidemia. She had been administered

Table 1 Clinical data and Brunt stage

\begin{tabular}{|c|c|c|c|c|c|c|c|c|}
\hline Case & Age/sex & $\mathrm{CD}$ & $\mathrm{DM}$ & BMI & $\begin{array}{l}\text { AST/ALT } \\
(\mathrm{IU} / \mathrm{l})\end{array}$ & $\begin{array}{l}\gamma \mathrm{GTP} \\
(\mathrm{IU} / \mathrm{l})\end{array}$ & $\begin{array}{l}\mathrm{TC} \\
(\mathrm{mg} / \mathrm{dl})\end{array}$ & Brunt stage \\
\hline 1 & $36 / \mathrm{M}$ & Liver injury & - & 26.8 & $83 / 186$ & 390 & 122 & 1 \\
\hline 2 & $37 / \mathrm{F}$ & Liver injury & - & 26.5 & $136 / 210$ & 70 & 229 & 3 \\
\hline 3 & $63 / \mathrm{F}$ & Liver injury & - & 25.4 & $67 / 112$ & 44 & 218 & 3 \\
\hline 4 & $37 / \mathrm{M}$ & Infarct of MO & Pre & 32.9 & $35 / 61$ & 152 & $102^{\mathrm{a}}$ & 3 \\
\hline
\end{tabular}

$C D$ clinical diagnosis, $D M$ diabetes mellitus, $B M I$ body mass index, $T C$ total cholesterol, infarct of $M O$ infarction of the medulla oblongata, pre prediabetes mellitus

${ }^{\text {a }}$ Low-density lipoprotein cholesterol 


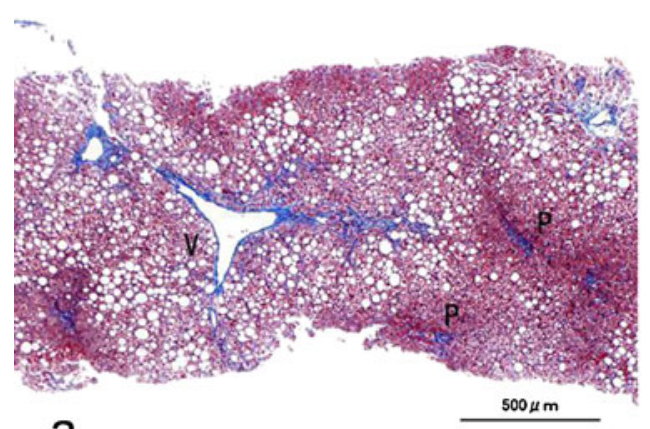

a

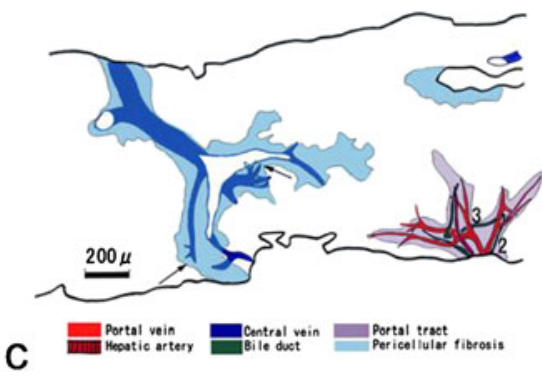

Fig. 1 Histology and histologic reconstruction of the first case. a Photomicrograph at a low magnification of the first reconstructed area. The lobular architecture is preserved. Macrovesicular steatosis of the liver cells is prominent with a centrilobular distribution (Masson's trichrome). b Photomicrograph at a low magnification of the second reconstructed area. The lobular architecture and portal tract are preserved, and macrovesicular steatosis is the same as in a (Masson's trichrome). c Histologic reconstruction of the first area. Pericellular fibrosis tends to expand more widely around the central vein and its

steroids for drug eruption for a short period 6 months earlier. She underwent liver biopsy on the suspicion of NASH.

Histological features were somewhat irregular with regard to centrilobular fibrosis (Fig. 2a, b). Although partial central to central pericellular bridging fibrosis had developed, the basic lobular structure was preserved. The portal tracts were slightly enlarged by fibrosis. Mild to moderate macrovesicular steatosis was found unevenly distributed in the lobules. Ballooning cells were scattered throughout the specimen. Necroinflammatory lesions and Mallory-Denk bodies were scarce.

Two regions were subjected to histological reconstruction. Each reconstruction comprised of 110 sections $(385 \mu \mathrm{m})$. The light microscopic features corresponding to the first reconstructed area are shown in Fig. 2a. The reconstruction is shown in Fig. 2c. Pericellular fibrosis spreads around the central vein and terminal portions more extensively than that in the first case. Characteristic features of these vessels were fibrous thickening of the wall and marked segmental stenosis of the lumen (phlebosclerosis) [arrows in Fig. $2 c(\alpha)$ ]. Some terminal portions were buried in the pericellular fibrosis. In part, mild pericellular fibrosis extended into the parenchyma in a band-like fashion [arrowheads in Fig. $2 c(\alpha)$ ]. Although

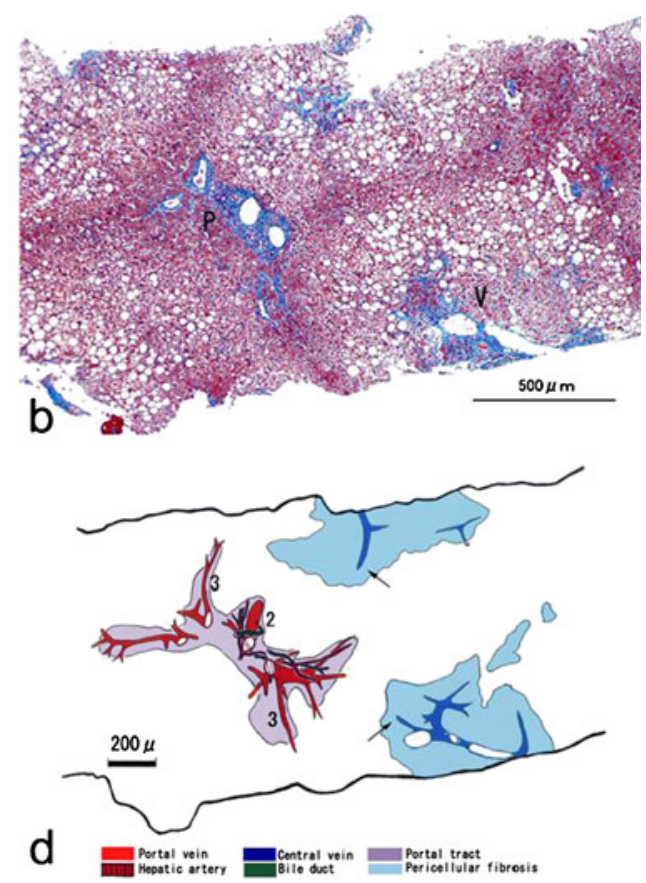

terminal portions than around the proximal portion (sublobular vein). Some distal portions are buried in the fibrotic area (arrows). The branching pattern of the portal vein remains almost normal in the portal tract. d Histologic reconstruction of the second area. The areas with pericellular fibrosis expand widely around the hepatic veins (central veins). Some terminal portions of the central vein are buried in the fibrotic area (arrows). a, b $P$ portal vein, $V$ central vein; c, d 2 second step branch, 3 third step branch

the number of portal veins was mostly preserved in portal tracts mildly enlarged through fibrosis, third step branches of which the normal branching pattern had been lost were noted to run parallel [Fig. $2 \mathrm{c}(\alpha, \beta)$, compare to Fig. 1c, d] consistent with beginning damage to the portal tract. However, the parenchyma was still reached by third step branches. A photomicrograph of the second reconstructed area is shown in Fig. 2b. The arrow in Fig. $2 b$ indicates delicate pericellular fibrosis partially changing to fibrous septa. The second reconstruction is shown in Fig. 2d. It was noteworthy that the pericellular fibrosis developing around the central vein and its terminal portions in one lobule extended and connected to a band-like or similar pattern of fibrosis in an adjacent lobule. On the other hand, the portal tract itself was only slightly enlarged with fibrosis and mild inflammation. Furthermore, the distribution of the portal trees was basically preserved, and third step branches broke up into the sinusoids.

The third case was a 63-year-old Japanese female who had suffered from hyperlipidemia and been treated with antihyperlipidemic drug for 3 years. Abnormal liver function was detected in a blood test during the course of the illness. She underwent liver biopsy because of deterioration of liver function as determined by the blood test. 

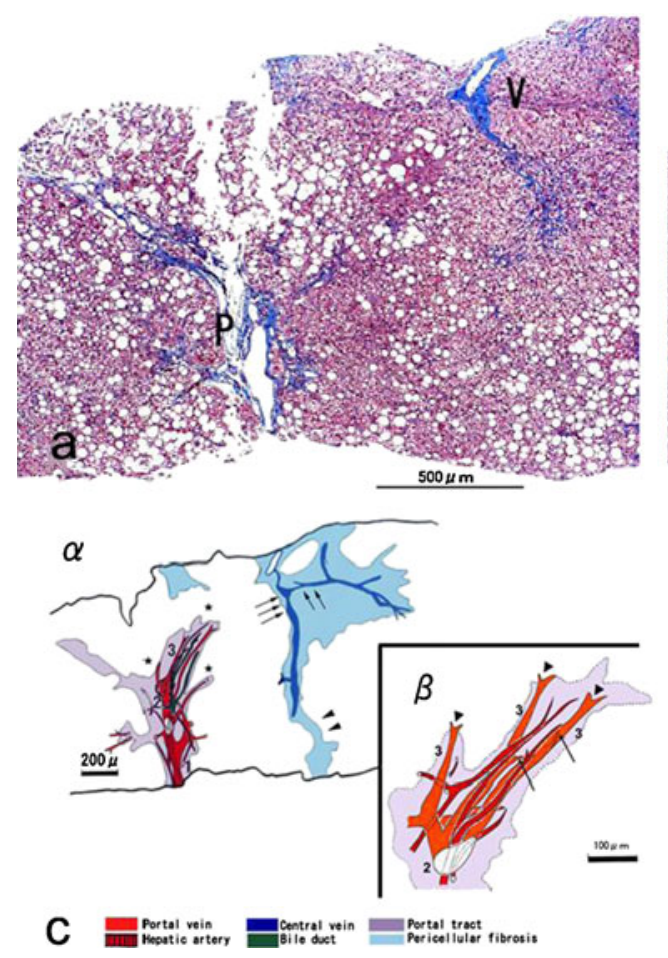

Fig. 2 Histology and histologic reconstruction of the second case. a Photomicrograph at a low magnification of the first reconstructed area. There is moderate macrovesicular steatosis. Pericellular fibrosis is seen in the upper right quadrant of the picture (Masson's trichrome). b Photomicrograph of the second reconstructed area. In contrast to the portal tract with good preservation, pericellular fibrosis and fibrosis are prominent along the central and sublobular veins. Delicate pericellular fibrosis changes partially to band-like fibrosis (arrow) (Masson's trichrome). $\mathbf{c}$ Histologic reconstruction of the first area. $\alpha$ Pericellular fibrosis develops widely around central veins and extends further into the parenchyma (arrowheads). Arrows indicate phlebosclerotic change

Histological features are shown in Fig. 3a, b. Fibrosis remarkably expanded around the central vein and central to central band-like fibrous bridging developed. In places, the fibrotic areas around the central veins were observed to be quite close to the portal tract. In contrast, the portal tracts were well preserved or slightly enlarged with fibrosis. Moderate to severe macrovesicular steatosis was found throughout the lobule. Mild inflammatory cell infiltration, some ballooning cells, and small arteries were found in the centrilobular areas with fibrosis (arrow in Fig. 3c).

The light microscopic features corresponding to the reconstructed area are shown in Fig. 3b. The histological reconstruction comprised of 93 sections $(325 \mu \mathrm{m})$ and is shown in Fig. 3d. Centrilobular fibrosis progressed more than in cases 1 and 2. The centrilobular fibrotic areas of adjoining lobules were apparently connected by band-like bridging fibrosis. As a result, band-like fibrosis incompletely surrounded portal tracts. In addition, this fibrosis was loosely connected to the portal tract (Fig. 3b, d). The terminal portions of the central vein in the fibrotic area on the
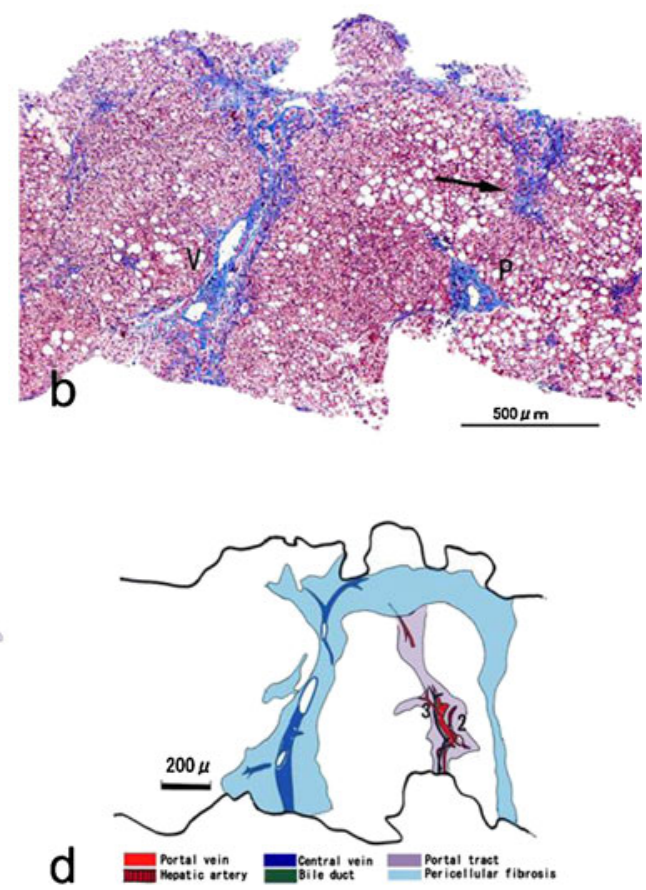

of the central vein. Third step branches of the portal vein run parallel with the loss of the normal branching pattern in the mildly enlarged portal tract with fibrosis. $\beta$ Histologic reconstruction of the area indicated by asterisks in $\mathbf{c}$ at a high magnification. The area was mildly fibrosed. Three third step branches run into the sinusoids (arrowheads). Some branches from third step branches are stenosed and disappeared (arrows). d Histologic reconstruction of the second area. Characteristic feature is that arch-shaped bridging fibrosis incompletely surrounds the portal tract. The bridging fibrosis is not connected with the fibrosis issued from the portal tract. a, b $P$ portal vein, $V$ central vein; c, d 2 second step branch, 3 third step branch

right side of the image had increased in number and ran randomly in a complex pattern. Small arteries had developed around veins in the fibrotic area. Although the portal vein of the parenchymal portion in the portal tracts was generally preserved and nourished the parenchyma, part of the second step branch of the portal vein showed a relatively sharp curve (asterisk in Fig. 3d). Although the slightly distorted angioarchitecture as well as the loose fibrous connection between the central vein and portal tract indicates the beginning of lobular restructuring, the basic lobular architecture was still preserved.

The fourth case was a 37-year-old male Japanese patient who took medication for schizophrenia. He also suffered from prediabetes mellitus, hyperlipidemia, and hypertension. He died suddenly a week after the onset of infarction of the medulla oblongata. An autopsy was carried out $1 \mathrm{~h}$ and $30 \mathrm{~min}$ after death.

Histological features are shown in Fig. 4a-c. Fibrosis around the central and sublobular veins had progressed remarkably, and band-like fibrous bridging had frequently 


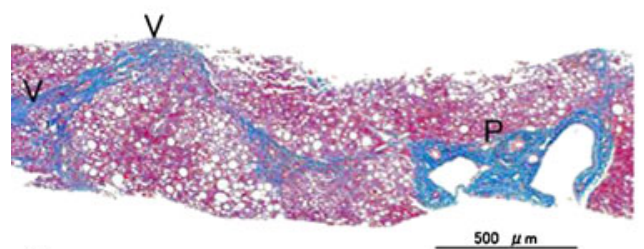

a

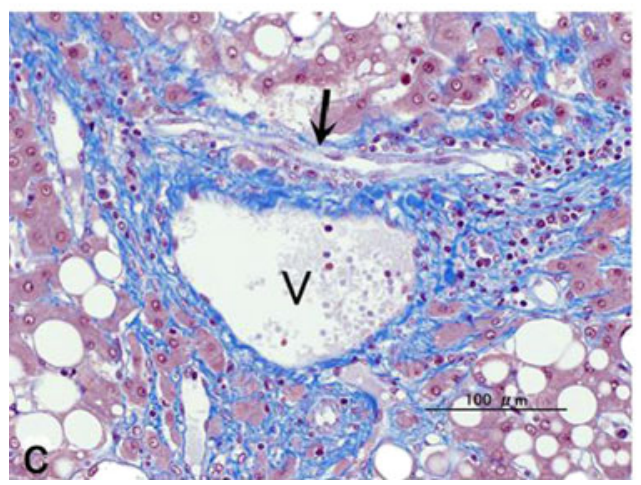

Fig. 3 Histology and histologic reconstruction of the third case. a Photomicrograph at a low magnification shows mild fatty liver, bridging fibrosis between the central veins. In addition, the central vein and the portal tract are close to each other (Masson's trichrome). b Photomicrograph of the reconstructed area. Fibrosis expands around the central veins and connects loosely between the central vein and portal tract (Masson's trichrome). c Arteries are recognized around the central

developed between the central veins in adjoining lobules. In addition, in some places, portal tracts were connected to band-like fibrotic areas. Although many portal tracts were normal, some distal portal tracts showed damage with a luminal narrowing of the portal vein, mild ductular proliferation, and mild fibrosis. Moderate macrovesicular steatosis was localized around the fibrotic areas. Ballooning cells and infiltrating inflammatory cells were scattered throughout the parenchyma.

Three regions were selected for histological reconstruction. The histological features corresponding to the first reconstructed area are shown in Fig. 4a. The histological reconstruction comprised of 160 sections $(560 \mu \mathrm{m})$ at a low magnification (Fig. 4d) and covered both conducting and parenchymal portions of portal veins. Fibrosis was usually found around the sublobular and central veins throughout the liver tissue. The fibrosis progressed to band-like fibrosis resulting in the formation of a network connecting the central veins and surrounding the distal portal tracts. A few portal tracts were connected to the fibrotic areas around the central veins (asterisks in Fig. 4d). However, it was difficult to distinguish between fibrosis issued from the central vein region and fibrosis from the portal tract. The branching pattern of the first and second step branches of the portal vein seemed to be generally preserved in most of the
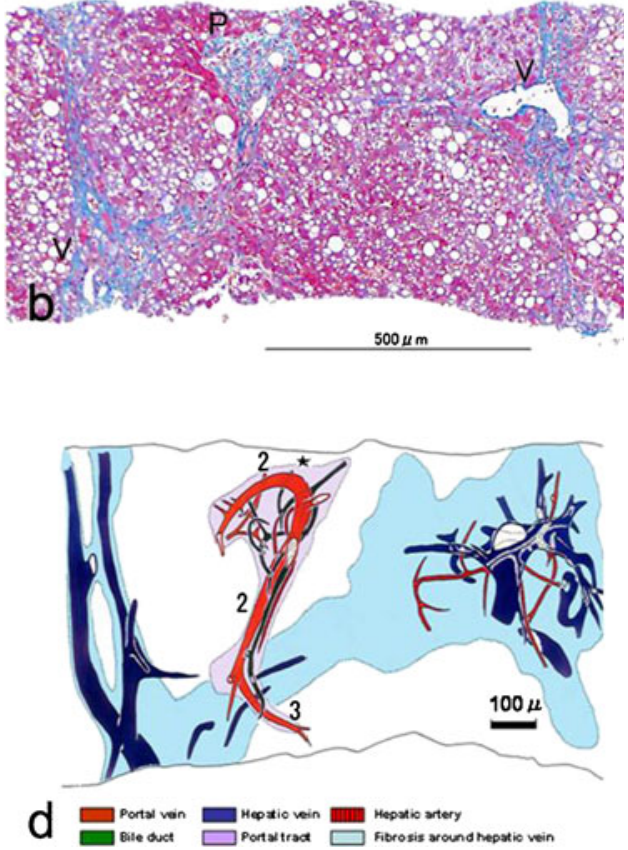

vein (arrow) (Masson's trichrome). d Histologic reconstruction. Fibrous bridging is recognized between central veins and incompletely surrounds the portal tract. Note the arteries developing around the central veins. In the portal tract, the second portion of the portal vein shows an abnormal abrupt bend (asterisk). a-c $P$ portal vein, $V$ central vein; $\mathbf{d} 2$ second step branch, 3 third step branch

parenchymal portion. Additionally, there was a very interesting feature in that arteries were well developed around the hepatic veins and apparently originated from preexisting arteries in the portal tracts (arrows in Fig. 4d).

The second histological reconstruction at moderate magnification comprised of 80 sections $(280 \mu \mathrm{m})$ and focused on central vein areas (Fig. 4e). The histological features corresponding to the reconstruction are shown in Fig. 4b. Arteries were twined around the central veins like ivy and showed anastomosis in places. Fibrous connection was found between the peripheral portal tract and fibrous band involving the central veins.

The histological features corresponding to the third histological reconstruction are shown in Fig. 4c. Figure $4 \mathrm{f}$ shows the reconstruction, which comprised of 80 sections $(280 \mu \mathrm{m})$, at a moderate magnification focusing mainly on the portal veins of the parenchymal portion. The branching pattern of the portal vein was well preserved. The third step branches in particular ran almost normally into the parenchyma to nourish hepatocytes. This indicates that the lobular architecture was almost undamaged. There was also abnormal arterial development around the sublobular hepatic vein and the fibrous connection between the portal tract and fibrously expansive area around the central vein. 

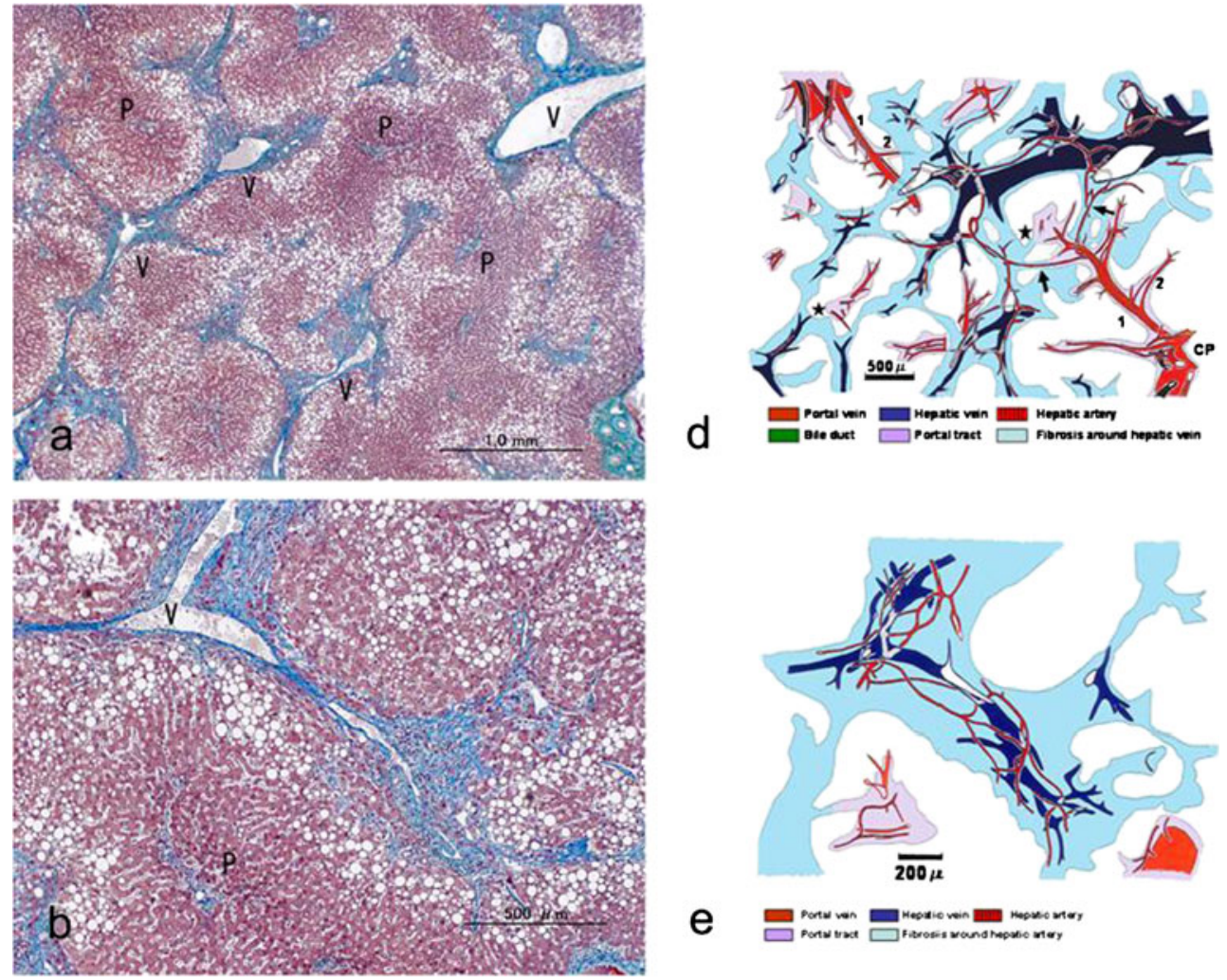

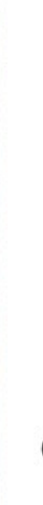

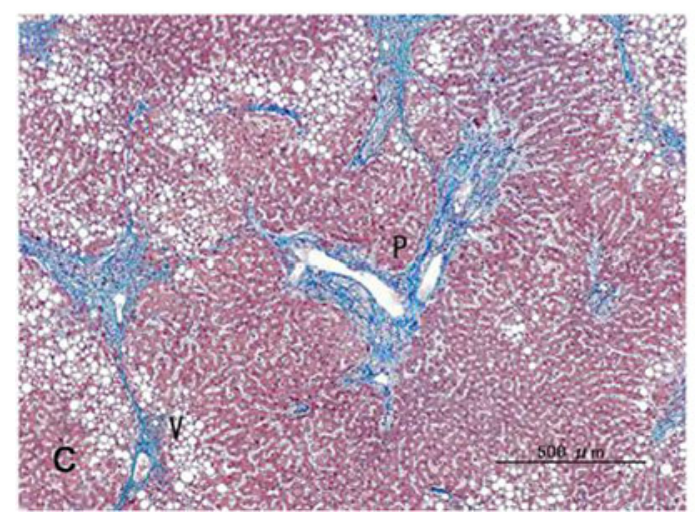

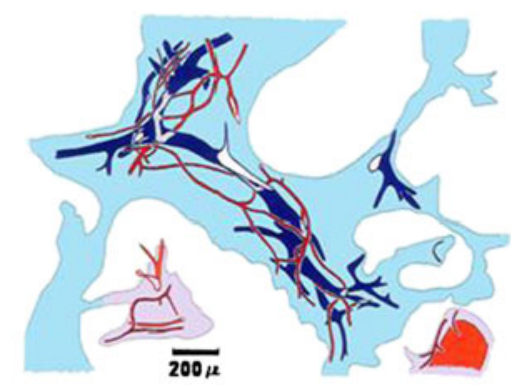

e
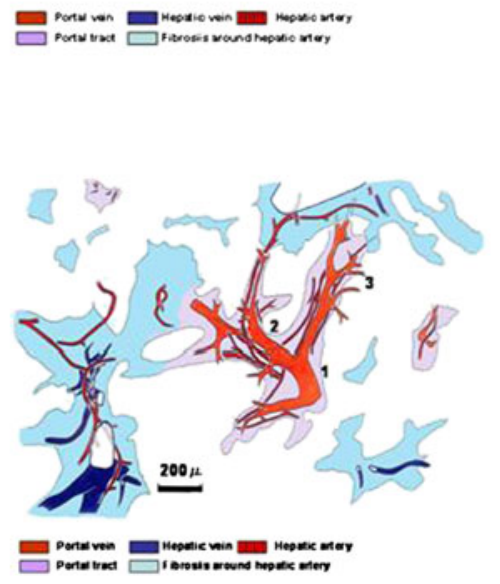

Fig. 4 Histology and histologic reconstruction of the fourth case. a-c Photomicrographs shown in a-c correspond to the histologic reconstructions shown in $\mathbf{d}-\mathbf{f}$, respectively. a A general view; $\mathbf{b}, \mathbf{c}$ views focused on the central vein and portal tract, respectively. Moderate steatosis is noted around the central vein with fibrosis. Bridging fibrosis is frequently found mainly between the central and hepatic veins. In contrast to the hepatic veins, the portal tracts show a tendency to be preserved (Masson's trichrome). d Histologic reconstruction with a general view at low magnification. Bile ducts are omitted except for large portal tracts. Sections of bridging fibrosis between the central and hepatic veins are connected together and finally form a fibrous meshwork. The portal tracts are located within the fibrous band mesh. In only a few places, the portal

\section{Discussion}

Ludwig et al. [4] coined the term nonalcoholic steatohepatitis in non-drinkers because the histological features are strikingly similar to those of alcoholic hepatitis. In the histological assessment of NASH, agreement among pathologists in terms tracts connect to the fibrotic area around the central veins (asterisks), although portal branches are generally preserved. Arteries run around the central and hepatic veins. The arteries originate from the interlobular arteries of the portal tracts (arrows). e The reconstructions are focused on central veins. Arteries run in a complex manner around the vein like ivy. $\mathbf{f}$ Histologic reconstruction is focused on the portal tract. Biliary trees are omitted. The branching pattern of the parenchymal portion of the portal vein is maintained, and the most distal branch (the third step branch) runs into the parenchyma to nourish hepatocytes. Arteries originating from the portal tract reach the fibrotic area around the hepatic vein. $\mathbf{a}-\mathbf{c} P$ portal vein, $V$ central vein; $\mathbf{d}-\mathbf{f} C P$ conducting portion of the portal vein; 1 first step branch, 2 second step branch, 3 third step branch

of grading steatosis and fibrosis is satisfactory, while the agreement in terms of grading inflammation and liver cell damage tends to be not $[18,21,22]$.

The pathogenesis of NASH has yet to be clarified, but as a response to steatosis, oxidative stress, endotoxin-mediated cytokines, and immune mechanisms are considered to be 
important, but also in alcoholic hepatitis [23]. Recently, Neuschwander-Tetri [24] proposed the lipotoxic liver injury hypothesis for the pathogenesis of NASH. This hypothesis suggests that metabolites of free fatty acids cause lipotoxic hepatocellular injury.

Taking into account that NASH can progress to cirrhosis, the morphological changes in the transition of steatohepatitis to cirrhosis should be elucidated to understand the pathophysiology accurately and to predict patient prognosis. According to Brunt et al. [17], zone 3 perisinusoidal/pericellular fibrosis develops first and is followed by portal fibrosis. In advanced stages, bridging fibrosis between the central veins or between central veins and portal tracts occurs, followed by cirrhosis. Kleiner et al. [18] modified the fibrosis staging in the Brunt system by dividing fibrosis scores for stage 1 into delicate (1a), dense perisinusoidal fibrosis (1b), and non-bridging portalonly fibrosis (1c). Stage 2 accounts for perisinusoidal and portal/periportal fibrosis. This is consistent with portal fibrosis beginning early in the disease process and playing an important role in connection with the development of portal-central fibrous bridging.

The first case in this study was stage $1 \mathrm{~b}$ according to Kleiner [18], and the other three cases were stage 3. Therefore, in the latter cases, it is not clear whether perisinusoidal or portal/periportal fibrosis preceded in the early stage. Taking into account that our study did not consider early portal fibrosis, we limit the discussion on the evolution of fibrosis to pericellular-only, pericellular with portal/periportal, and bridging fibroses.

In this study, we undertook a detailed three-dimensional investigation of the morphogenesis of the structural changes of NASH at the non-cirrhotic stage and observed how fibrosis progressed in connection with restructuring of the liver lobule as associated with the changing angioarchitecture. With regard to the evolution of fibrosis, the most characteristic feature which occurred in all our cases was pericellular fibrosis around the central vein and its terminal portions. Pericellular fibrosis evolved in mid and central zones of the classical lobule, in which the former terminal portions are distributed, and tended to change gradually into dense fibrosis, also called septal fibrosis, with loss of enmeshed hepatocytes. During this process, the central vein tended to show phlebosclerosis with a thickened wall and narrowing of the lumen, while pericellular fibrosis occurred in a band-like fashion with or without dense fibrosis extending further into the lobule. Subsequently, the band-like fibrosis in a one lobule gradually connected to a similar lesion in the adjoining lobule. The latter event reflects interruption of the lobular parenchyma. Band-like fibrosis surrounding the portal tract was archlike in shape at a relatively early stage and formed an imperfect or perfect ring with the evolution of fibrosis, as seen in the fourth case. Fibrosis developed along the central vein and its terminal portions and extended toward halfway the interportal distance.
Consequently, fibrosis arising from adjoining lobules connects halfway the interportal distance and forms an archshaped bridge (the central to central fibrous bridge). This fibrotic bridge corresponds to the microcirculatory periphery, a zone of potentially low lobular blood flow. The geographical distribution of fibrosis in NASH reflects congestive bridges between centrilobular areas in advanced chronic congestion [25] as seen in cardiac cirrhosis [26]. In our observations, although portal tracts showed a tendency to be enlarged by fibrosis, the severity of fibrosis was generally mild. However, some portal tracts were close or adherent to the loose or bandlike fibrosis arising from the fibrotic area around the central vein. It is noteworthy that arteries originating from preexisting arteries in the portal tract often developed around veins in the non-cirrhotic stage [27]. It is conceivable that arterial development is inevitable in the process of fibrosis in view of the parallel evolution of progressive fibrosis and the development of arterial branches around central veins. In the non-cirrhotic stage of NASH, portal veins in the portal tracts themselves tended to be preserved, in contrast to the centrilobular areas. Because the skeleton of the lobular architecture is essentially formed by the parenchymal portion of the portal veins, the lobular architecture tends to be maintained, as seen in our fourth case and the reason why our cases were judged to be in the non-cirrhotic stage. This sharply contrasts to the cirrhotic stage in terms of the remarkable alteration of the angioarchitecture, especially of that of the portal vein $[28,29]$.

It is suggested that the formation of the fibrous band between the central veins is an early event in the process of restructuring of the lobule in NASH. Subsequently, the portal tracts are surmised to be gradually damaged and enlarged with fibrosis, which is accompanied by various pathologic changes of the portal veins. Distortion of the liver lobule might be expected in the subsequent stage when the fibrous bridging has formed between the portal tracts and between the portal tracts and the central veins throughout the liver, in association with the distortion of the angioarchitecture of the portal vein. We would like to stress that there is a characteristic stage between Brunt stages 2 and 3 in which central to central fibrous bridging is remarkably dominant.

In cases of chronic viral hepatitis, the portal tract damage with pathologic changes of the portal vein generally precedes central vein involvement. Subsequently, bridging fibrosis between portal tracts and between portal tracts and central veins occurs relatively early in the process of restructuring of the lobules [30]. Therefore, it is assumed that NASH and chronic viral hepatitis progress to cirrhosis along different pathways.

To verify the whole process of restructuring with fibrosis and to strengthen our conclusion, further observation of more cases of NASH with histologic reconstruction will be necessary. 
Acknowledgments We thank the staff members of the Department of Pathology, Jikei University School of Medicine, for their technical assistance.

Conflict of interest The authors state that they have nothing to declare regarding funding or a conflict of interest with respect to this manuscript.

Open Access This article is distributed under the terms of the Creative Commons Attribution License which permits any use, distribution, and reproduction in any medium, provided the original author(s) and the source are credited.

\section{References}

1. Adler M, Schaffner F (1979) Fatty liver hepatitis and cirrhosis in obese patients. Am J Med 67:811-816

2. Falchuk KR, Fiske SC, Haggitt RC, Federman M, Trey C (1980) Pericentral hepatic fibrosis and intracellular hyaline in diabetes mellitus. Gastroenterology 78:535-541

3. Lee RG (1989) Non alcoholic steatohepatitis: a study of 49 patients. Hum Pathol 20:594-598

4. Ludwig J, Viggiano TR, McGill DB, Otto BJ (1980) Nonalcoholic steatohepatitis. Mayo clinic experience with hitherto unnamed disease. Mayo Clin Proc 55:434-438

5. Nagore N, Scheuer PJ (1988) The pathology of diabetic hepatitis. J Pathol 156:155-160

6. Poewll EE, Graham W, Cooksley E, Hanson R, Searle J, Halliday JW, Powell LW (1990) The natural history of nonalcoholic steatohepatitis: a follow-up study of forty-two patients for up to 21 years. Hepatology 11:74-80

7. Adler M, Schaffner F (1990) Fatty liver hepatitis (steatohepatitis) and obesity: an autopsy study with analysis of risk factors. Hepatology 12:1106-1110

8. Chitturi S, Abeygunasekera S, Farrell GC, Holms-Walker J, Hui JM, Hui JM, Fung G, Kari R, Lin R, Samarasinghe D, Liddle G, Weltman M, George J (2002) NASH and insulin resistance: insulin hypersecretion and specific association with the insulin resistance syndrome. Hepatology 35:373-379

9. Gastaldelli A, Harrison SA, Bekfort-Aguilar R, Hardies LJ, Balas B, Schenker S, Cusi K (2009) Importance of changes in adipose tissue insulin resistance to histological response during thiazolidinedione treatment of patients with nonalcoholic steatohepatitis. Hepatology 50:1087-1093

10. Angulo P (2002) Nonalcoholic fatty liver disease. N Engl J Med 346:1221-1231

11. Brunt EM (2004) Nonalcoholic steatohepatitis. Semin Liver Dis 24:3-20

12. Reid AE (2001) Nonalcoholic steatohepatitis. Gastroenterology 121:710-723

13. Ludwig J, McGill D, Lindor KD (1997) Nonalcoholic steatohepatitis. J Gastroenterol Hepatol 12:398-403

14. McCullough AJ (2002) Update on nonalcoholic fatty liver disease. J Clin Gastroenterol 34:255-262
15. Sanyal AJ (2002) AGA technical review on nonalcoholic fatty liver disease. Gastroenterology 123:1705-1725

16. Lefkowitch JH, Morawski JL (2012) Late nonalcoholic fatty liver disease with cirrhosis: a pathologic case of lost or mistaken identity. Semin Liver Dis 32:92-98

17. Brunt EM, Janney CG, Bisceglie AMD, Neuschwander-Tetri BA, Bacon BR (1999) Nonalcoholic steatohepatitis: a proposal for grading and staging the histological lesions. Am J Gastroenterol 94:2467-2474

18. Kleiner DE, Brunt ME, Van Natta M, Behling C, Contos MJ, Cummings OW, Ferell LD, Liy Y-C, Torbenson MS, UnalpArida A, Yeh M, McCullough AJ, Sanyal AJ (2005) Design and validation of histological scoring system for nonalcoholic fatty liver disease. Hepatology 41:1313-1321

19. Matsumoto T, Kawakami M (1982) The unit concept of hepatic parenchyma - a reexamination based on angioarchitectural studies. Acta Pathol Jpn 32:285-314

20. Crawford JM, Burt AD (2012) Anatomy, pathophysiology, and basic mechanism of disease. In: Burt A, Portmann B, Ferrell (eds) Pathology of the liver. Churchill Livingstone, Edinburgh, pp $1-78$

21. Fukusato T, Fukushima J, Shiga J, Takahashi Y, Nakano T, Maeyama S, Uchikoshi M, Ohbu M, Matsumoto T, Matsumoto K, Hano H, Sakamoto M, Kondo F, Komatsu A, Ishikawa T, Ohtake H, Takikawa H, Yoshimura K, Liver disease working Group-Kanto (2005) Interobserver variation in the histopathological assessment of nonalcoholic steatohepatitis. Hepatol Res $33: 122-127$

22. Younossi ZM, Gramlich T, Lu YC, Matteoni C, Petrelli M, Goldblum J, Rybicki L, McCullough AJ (1998) Nonalcoholic fatty liver disease: assessment of variability in pathologic interpretations. Mod Pathol 11:560-565

23. Day CP, Saksena S (2002) Non-alcoholic hepatitis: definition and pathogenesis. J Gastroenterol Hepatol 17:S377-S384

24. Neuschwander-Tetri BA (2010) Hepatic lipotoxicity and the pathogenesis of nonalcoholic steatohepatitis: the central role of nontriglyceride fatty acid metabolites. Hepatology 52:774788

25. Lefkowitch JH, Mendez R (1986) Morphologic features of hepatic injury in cardiac disease and shock. J Hepatol 2:313-327

26. Rosenberg PM, Friedman LS (2003) The liver in circulatory failure. In: Schiff ER, Sorrell MF, Maddrey WC (eds) Schiff's disease of the liver. Lippincott Williams \& Wilkins, Philadelphia, pp $1327-1340$

27. Gill RM, Wilson L, Bass NM, Ferrell LD (2011) Centrizonal arteries and microvessels in nonalcoholic steatohepatitis. Am J Surg Pathol 35:1400-1404

28. Hano H (1979) Three dimensional approach to the angioarchitecture in a cirrhotic liver of alcoholic origin. Jikeilai Med J 94:1-12

29. Hayashi H (1989) Angioarchitectural changes in a mixed nodular cirrhotic liver. Acta Pathologica Jpn 30:1687-1697

30. Hano H, Takasaki S (2003) Three-dimensional observations on the alterations of lobular architecture in chronic hepatitis with special reference to its angioarchitecture for a better understanding of the formal pathogenesis of liver cirrhosis. Virchows Arch 443:655663 